\title{
Strong correlation between field-induced magnetism and superconductivity in $\operatorname{Pr}_{0.89} \mathrm{LaCe}_{0.11} \mathrm{CuO}_{4}$
}

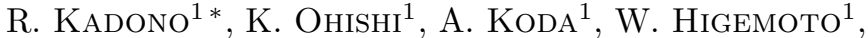 \\ K. M. Kojima ${ }^{2}$, M. Fujita ${ }^{3}$, S. Kuroshima ${ }^{3}$ and K. Yamada ${ }^{3}$ \\ ${ }^{1}$ Institute of Materials Structure Science, High Energy Accelerator Research Organization (KEK), Tsukuba, Ibaraki 305-0801 \\ ${ }^{2}$ Department of Physics, University of Tokyo, Tokyo 113-8656, Japan \\ ${ }^{3}$ Institute for Chemical Research, Kyoto University, Uji, Kyoto 610-0011
}

(Received July 8, 2004)

\begin{abstract}
We report the local magnetic response of an electron-doped cuprate superconductor, $\operatorname{Pr}_{0.89} \mathrm{LaCe}_{0.11} \mathrm{CuO}_{4-y}\left(T_{c} \simeq 26 \mathrm{~K}\right)$, studied by muon spin rotation $(\mu \mathrm{SR})$ over a field range from 200 Oe to $50 \mathrm{kOe}$. In the paramagnetic state, the muon Knight shift along the crystal $c$-axis $\left(K_{\mu}^{z}\right)$ is proportional to the in-plane susceptibility $\chi_{a b}$. More surprisingly, $K_{\mu}^{z}$ is strongly enhanced by the occurrence of superconductivity (below $\sim 40 \mathrm{kOe}$ ), changing its sign from positive to negative around $\sim 1 \mathrm{kOe}$ with increasing field. This finding can be understood by considering the weak polarization of Pr ions due to superexchange interaction with the antiferromagnetic $\mathrm{Cu}$ sublattice, which coexists with superconductivity.
\end{abstract}

KEYWORDS: electron-doped cuprates, superconductivity, magnetism, $\mu \mathrm{SR}$,

The role of antiferromagnetic (AF) correlation in the mechanism of superconductivity has been a subject of vigorous debate in the field of high- $T_{c}$ cuprate superconductors since their discovery and subsequent revelation of non-Fermi liquid behavior in hole-doped ( $p$ type) cuprates. Despite the common view that AF correlation is vital for the occurrence of superconductivity, opinions are largely divided as to the ground state with which the pair correlation is at work; namely, the one that can be mapped onto a conventional Fermi liquid, ${ }^{1}$ or a new state of the matter consisting of "spinons/holons" ${ }^{2}$ or a texture of self-organized carriers such as "stripes". ${ }^{3}$ Recent observation of field-induced magnetism in $p$ type cuprates has shed new light on this issue, where the recovery of the quasistatic AF state under a moderate magnetic field (a few Tesla) is suggested in the flux line lattice (FLL) state of $\mathrm{La}_{2-x} \mathrm{Sr}_{x} \mathrm{CuO}_{4}$ (LSCO), ${ }^{4-6}$ $\mathrm{YBa}_{2} \mathrm{Cu}_{3} \mathrm{O}_{7-\delta} \quad(\mathrm{YBCO}){ }^{7}{ }^{7} \mathrm{YBa}_{2} \mathrm{Cu}_{4} \mathrm{O}_{8} \quad\left(\mathrm{Y}_{1248}\right),{ }^{8}$ and $\mathrm{Bi}_{2} \mathrm{Sr}_{2} \mathrm{CaCu}_{2} \mathrm{O}_{8+\delta}$ (BSCCO). ${ }^{9}$ Since the superconducting order parameter is locally suppressed in the vortex cores, there is a possibility that these findings may be a manifestation of the electronic ground state nucleated in the normal cores. However, while all of the observed effects are attributed to the AF (or quasistatic stripe) phase localized in the vortex cores, the evidence for the "AF core" remains elusive; the results in LSCO are all from neutron diffraction measurements which are not sensitive to the local structure over such a large length scale (vortices with a core size of $\sim 10^{2} \AA$, separated by $10^{2}-10^{3} \AA$ ), those in YBCO and $\mathrm{Y}_{1248}$ are based on NMR measurements where the evidence is an enhancement of spin-lattice relaxation near the core rather than a welldefined shift, and that for BSCCO is from STM where the measured quantity is subject to the strong anisotropy of tunnel conductance. One attempt to directly observe modified field distribution near the vortex cores in YBCO $(\delta=0.5)$ by the muon spin rotation $(\mu \mathrm{SR})$ technique yielded only a marginal sign of such modulation. ${ }^{10}$

Meanwhile, there is increasing evidence that the ground state of electron-doped ( $n$ type) cuprates may be understood within the framework of a conventional Fermi liquid. The normal state of $n$ type cuprates exhibits a quadratic temperature dependence $\left(\propto T^{2}\right)$ of the resistivity in contrast to the linear dependence seen in $p$ type cuprates. A recent NMR study of an $n$ type cuprate, $\mathrm{Pr}_{1-x} \mathrm{LaCe}_{x} \mathrm{CuO}_{4-y}$ (PLCCO, $\left.x=0.09\right)$, has demonstrated that the Korringa law is restored upon the removal of superconductivity by applying an external magnetic field above the upper critical field $\left(H_{c 2} \simeq 50\right.$ $\mathrm{kOe}) .{ }^{11}$ These are typical features found in the Fermi liquid ground state. Moreover, the spin dynamics in the superconducting state of an $n$ type cuprate exhibits commensurate spin fluctuation, ${ }^{12}$ whereas incommensurate spin fluctuation is commonly found in $p$ type cuprates. In these circumstances, the field-induced $\mathrm{AF}$ state recently observed in $\mathrm{Nd}_{1.85} \mathrm{Ce}_{0.15} \mathrm{CuO}_{4}$ is attracting much interest; ${ }^{13,14}$ it would be crucially important to examine whether or not such AF phase is localized within the vortex cores.

Recently, a much stranger external field effect in $\mathrm{Pr}_{2-x} \mathrm{Ce}_{x} \mathrm{CuO}_{4-y}$ (PCCO) has been observed by $\mu \mathrm{SR}$, where large copper moments $\left(\sim 0.4 \mu_{B}\right)$ are reported to have been induced by a magnetic field as small as 90 Oe. ${ }^{15} \mathrm{In}$ this paper, we report $\mu \mathrm{SR}$ measurements on the single crystalline $n$ type cuprate $\operatorname{Pr}_{0.89} \mathrm{LaCe}_{0.11} \mathrm{CuO}_{4-y}$ $\left(T_{c} \simeq 26 \mathrm{~K}\right)$, which is relatively close to the AF phase $(x \leq 0.1) \cdot{ }^{16}$ Compared with PCCO, we can obtain single crystals of PLCCO in much larger dimensions, which allows systematic studies by various experimental techniques including neutron diffraction. ${ }^{17}$ We show that the muon Knight shift, $K_{\mu}^{z}$, under an external magnetic field parallel to the $c$-axis is significantly influenced by the inplane susceptibility, $\chi_{a b}$, strongly suggesting the presence of unconventional hyperfine interaction involving a non- 
diagonal Fermi contact-type term between muons and Pr ions. Moreover, an additional shift of the frequency (corresponding to $\sim 10^{1} \mathrm{Oe}$ ) is induced spontaneously below $\sim T_{c}$ at lower fields $(<40 \mathrm{kOe})$ with either a positive or negative sign, depending on the magnitude of the applied magnetic field. This indicates that the in-plane polarization of Pr ions is clearly enhanced in the superconducting phase, strongly suggesting the influence of $\mathrm{CuO}_{2}$ planes on the behavior of Pr moments. Considering the field-induced weak antiferromagnetism of $\mathrm{CuO}_{2}$ planes in PLCCO ${ }^{17}$ we discuss the superexchange interaction between $\mathrm{Pr}$ and $\mathrm{Cu}$ moments as an origin of $\mathrm{Pr}$ moment polarization.
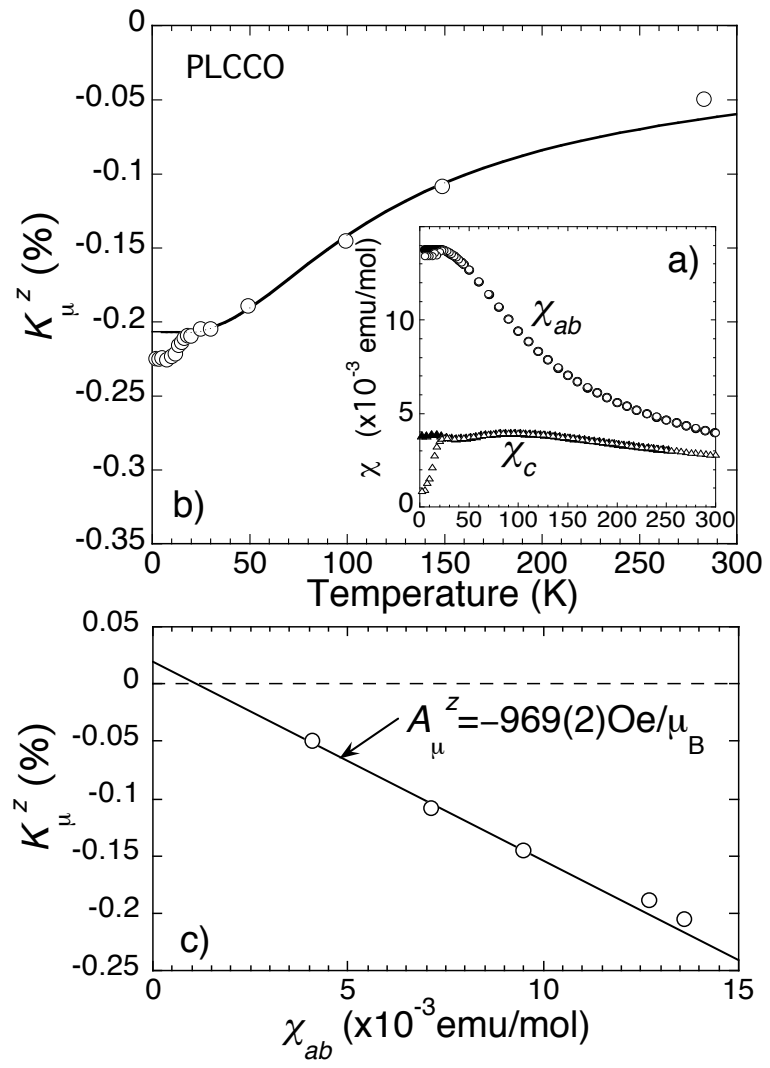

Fig. 1. (a) Temperature dependence of magnetic susceptibility at $H=20 \mathrm{kOe}$ (open symbols) and $50 \mathrm{kOe}$ (filled symbols) applied parallel $\left(\chi_{c}\right)$ and perpendicular $\left(\chi_{a b}\right)$ to the $c$-axis. (b) The muon Knight shift with $H=20 \mathrm{kOe}$ parallel to the $c$-axis, where the solid curve in (b) is proportional to $\chi_{a b}$. (c) The $K$-chi plot (with $\chi=\chi_{a b}$ ) for the Knight shift shown in (b).

A large single crystal of $\operatorname{Pr}_{0.89} \mathrm{LaCe}_{0.11} \mathrm{CuO}_{4-y}(y \simeq$ 0.02 ) was prepared by the traveling-solvent float zone method, the details of which have already been published elsewhere. ${ }^{16}$ A large volume fraction and the sharp onset of Meissner diamagnetism at $T_{c}$ (see below) demonstrated the high quality of the specimen. The $\mu \mathrm{SR}$ measurements were carried out on the M15 beamline at TRIUMF, Canada. A slab of $\mathrm{Pr}_{0.89} \mathrm{LaCe}_{0.11} \mathrm{CuO}_{4-y}$ crystal (measuring about $5 \mathrm{~mm} \times 8 \mathrm{~mm} \times 0.5 \mathrm{~mm}$ ) with the $c$-axis perpendicular to the plane was loaded onto a He gas-flow cryostat and a magnetic field $\left(\mathbf{H}=\left(0,0, H_{z}\right)\right)$ was applied parallel to the $c$-axis (where $z \| c$ ). In a transverse field (TF) geometry, the initial muon polarization was perpendicular to the $c$-axis so that the muon probed the local field $B_{z}$ by spin precession at a frequency $\gamma_{\mu} B_{z}$ (with $\gamma_{\mu}=13.553 \mathrm{MHz} / \mathrm{kOe}$ being the muon gyromagnetic ratio). Detailed zero-field (ZF) $\mu \mathrm{SR}$ measurements on the same specimen with varied oxygen depletion indicated a weak random magnetism similar to PCCO, which is identified as being due to the small Pr moments. ${ }^{18}$

The muon hyperfine parameter $A_{\mu}^{z}$ is deduced from a comparison between magnetic susceptibility $\chi$ and the muon Knight shift in the normal state; their relation in rare-earth metallic compounds is generally expressed as

$$
K_{\mu}^{z} \simeq K_{0}+\left(A_{c}+\sum_{i} A_{i}^{z z}\right) \chi_{c},
$$

where $K_{0}$ and $A_{c}$ denote the respective contributions from the $T$-independent Pauli paramagnetism and from the polarization of conduction electrons by the Rudermann-Kittel-Kasuya-Yoshida (RKKY) interaction, $\chi_{c}$ being the susceptibility for the normal directions, and $A_{i}^{z z}$ is the relevant component of the dipole tensor $A_{i}^{\alpha \beta}=\left(3 r_{i}^{\alpha} r_{i}^{\beta} / r_{i}^{2}-\delta_{\alpha \beta}\right) / r_{i}^{3}$ calculated for the nearby $i$-th $\operatorname{Pr}$ ions at a distance $\mathbf{r}_{i}$ from the muon. As shown in Fig. 1a, PLCCO exhibits a large anisotropy of $\chi$ between the in-plane $\left(\chi_{a b}\right)$ and normal $\left(\chi_{c}\right)$ directions, where $\chi_{a b}$ exhibits a significant increase with decreasing temperature while $\chi_{c}$ remains almost unchanged. The corresponding muon Knight shift versus temperature is shown in Fig. 1b; it is clear in Figs. 1a and 1b that $K_{\mu}^{z}$ is mostly proportional to $\chi_{a b}$, which is in a stark contrast to Eq. (1). Moreover, it is inferred from Fourier analysis of the TF- $\mu \mathrm{SR}$ spectra that there are two satellite peaks symmetrical about the central peak with about a half intensity whose splitting becomes large enough to be resolved above $\sim 25 \mathrm{kOe} .{ }^{18}$ Provided that the rare-earth sites are randomly occupied by $\mathrm{Pr}$ and La ions, the observed spectral pattern suggests a binomial distribution of hyperfine coupling constants.

Considering the earlier reports that the muon site is crystallographically unique and located near the oxygen atoms midway between the $\mathrm{CuO}_{2}$ planes $(z=0.25$ or 0.75 in the unit cell), ${ }^{19,20}$ we carried out extensive simulations of $K_{\mu}^{z}$ using Eq. (1). As a result, we found that there is no possibility for Eq. (1) to reproduce the welldefined three-frequency structure with binomial intensity distribution observed at higher fields. This is also obvious from the fact that the temperature dependence of $K_{\mu}^{z}$ is quite different from that of $\chi_{c}$. Thus, we need to introduce a hypothetical non-diagonal hyperfine interaction, $A_{f}$, to account for the observed result; namely,

$$
K_{\mu}^{z} \simeq K_{0}+A_{f} \chi_{a b} .
$$

Our result in Fig. 1b indicates that the term proportional to $\chi_{c}$ is negligible. (Note that the dipolar tensor $\sum A_{i}^{z z}$ actually takes null value when muons are sitting exactly at the center between two rare-earth ions.) The $K$-chi plot for $\chi_{a b}$ in Fig. 1c exhibits a linear relation with a small offset near the origin, from which we obtain $A_{f}=$ $A_{\mu}^{z}=-969(2)$ Oe $/ \mu_{B}$ for the central peak, and $-401(2)$ $\mathrm{Oe} / \mu_{B}$ and $-1551(2) \mathrm{Oe} / \mu_{B}$ for the satellites assuming a common $K_{0}(=201(3) \mathrm{ppm})$. Although the origin of $A_{f}$ 

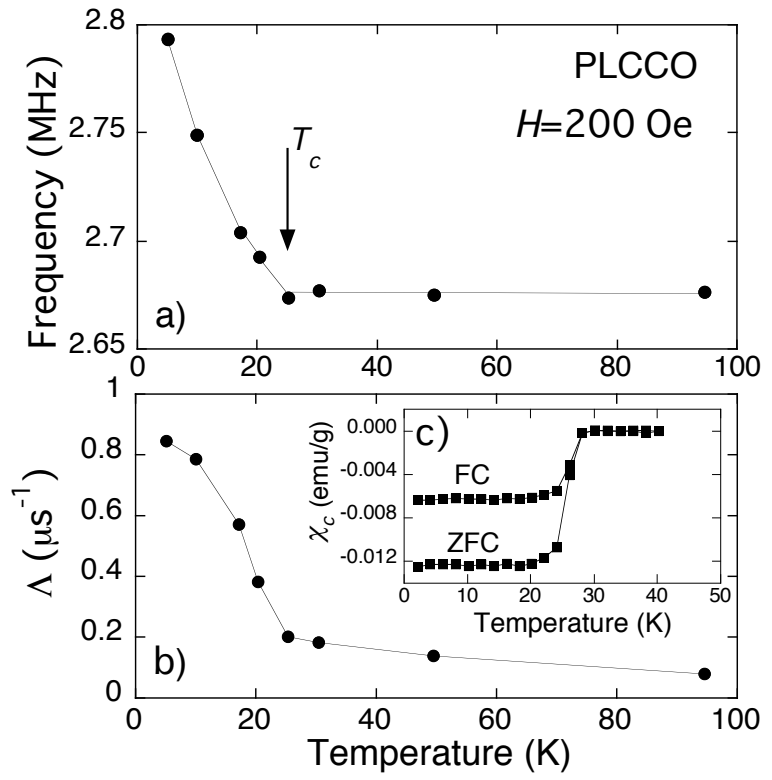

Fig. 2. Temperature dependence of the muon precession frequency (a) and transverse spin relaxation rate (b) at $H=200$ Oe. The inset (c) shows the Meissner effect observed in bulk susceptibility at 10 Oe under FC and ZFC conditions. The solid lines are a visual guide only.

is not clear at this stage, it is most probable that a Fermi contact-type (scalar) interaction (a "variation" of $A_{c}$ ) is responsible for the mechanism explaining the binomial distribution of the spectral weight.

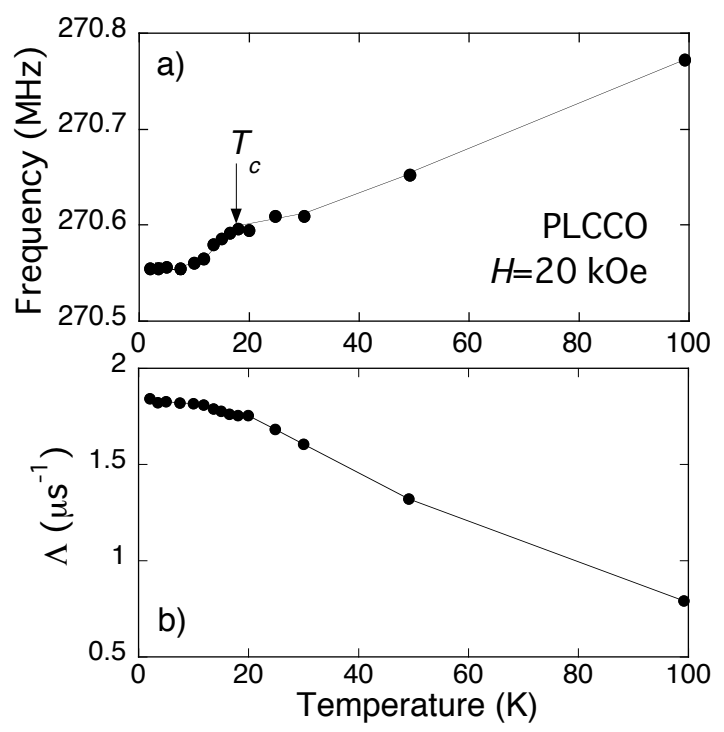

Fig. 3. Temperature dependence of the muon precession frequency (a) and transverse spin relaxation rate (b) at $H=20$ $\mathrm{kOe}\left(\right.$ where $\left.T_{c} \simeq 18 \mathrm{~K}\right)$. The solid lines are a visual guide only.

At the superconducting transition, the specimen exhibits a further peculiar magnetic response. As shown in Fig. 2, a large positive shift of the frequency and associated increase of the muon spin relaxation rate $(\Lambda)$ is observed at $H=200 \mathrm{Oe}$. While the increase of $\Lambda$ is understood by considering the inhomogeneous magnetic field distribution due to the FLL formation, the direction of the frequency shift is apparently opposite to that expected for diamagnetism in the FLL state. As shown in Fig. 2c, the possibility of attributing the observed positive shift to the so-called paramagnetic Meissner effect is ruled out by the magnetic response observed in both the ZFC and FC magnetization. This result is similar to what has been observed in PCCO. ${ }^{15}$ In Fig. 3, on the other hand, an enhancement of the frequency shift in the negative direction is observed below $T_{c}$ at $20 \mathrm{kOe}$.

The field dependence of this additional shift, $\Delta B_{z}=$ $B_{0}^{S}-B_{0}^{N}$ (frequencies divided by $\gamma_{\mu}$ ), is shown in Fig. 4, where $\Delta B_{z}$ exhibits a steep decrease with increasing field to change its sign to negative above $\sim 1 \mathrm{kOe}$. The strong field dependence at such low fields indicates that the shift of the Van Hove singularity due to the FLL formation is negligible over the entire field range; this is also consistent with a large magnetic penetration depth $(\geq 3400$ $\AA$ ) reported for PCCO. While the observed tendency is close to that in PCCO $(H \leq 2 \mathrm{kOe}),{ }^{15}$ the change in the sign of $\Delta B_{z}$ suggests the flip of $A_{\mu}^{z}$ due to the external field. (Unfortunately, $K_{\mu}^{z}$ in the normal state is too small to measure at such low fields.) Meanwhile, $\Delta B_{z}$ is only weakly dependent on the field for 2-40 KOe, above which it exhibits a trend to increase further in the negative direction. Such a behavior cannot be explained by the simple bulk demagnetization effect. More interestingly, these features above $\sim 2 \mathrm{kOe}$ (including a further increase of $\Delta B_{z}$ above $\sim 40 \mathrm{kOe}$ ) are quite similar to those of fieldinduced moments detected by neutron diffraction. ${ }^{17}$ The possibility to attribute the field-induced effect to impurity phases $(\mathrm{Pr}, \mathrm{Ce}, \mathrm{La})_{2} \mathrm{O}_{3}$ has been ruled out by the recent neutron experiment, as they found no such effect in those phases below $70 \mathrm{kOe} .^{14}$

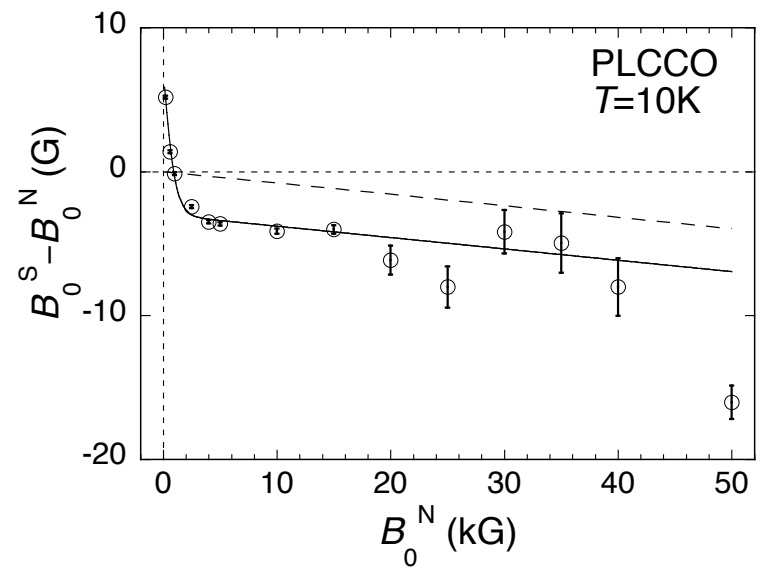

Fig. 4. Magnetic field dependence of the additional shift in the superconducting state, where $B_{0}^{S}$ and $B_{0}^{N}$ correspond to the internal field at $10 \mathrm{~K}$ and $30-40 \mathrm{~K}$, respectively. The solid curve is a "guide for the eye" fitting result by a phenomenological model assuming the flip of an internal field (with the dashed line representing the contribution of residual demagnetization).

Since the emergence of $\Delta B_{z}$ is strongly correlated with the occurrence of superconductivity below $\sim 40 \mathrm{kOe}$, the origin of $\Delta B_{z}$ must be in close relation with the electronic 
state of $\mathrm{CuO}_{2}$ planes. However, it has been demonstrated by very recent neutron diffraction measurements on the present specimen that the moment size of $\mathrm{Cu}$ ions induced by the external field is far smaller than that found in the $\mathrm{AF}$ phase of $\mathrm{Pr}_{1-x} \mathrm{LaCe}_{x} \mathrm{CuO}_{4-y}(x<0.1)$ with a quite non-linear dependence on the field; it remains almost constant over a range from 0 to $\sim 40 \mathrm{kOe} .{ }^{17}$ Considering the hyperfine coupling between $\mathrm{Cu}$ ions and muons at the relevant site (which is usually dominated by magnetic dipolar interaction), it is unlikely that such small $\mathrm{Cu}$ moments directly contribute to $\Delta B_{z}$, in contrast to what has been suggested for the case of PCCO. ${ }^{15}$ On the other hand, there is a strong superexchange coupling between $\mathrm{Cu}$ and $\mathrm{Pr}$ ions as inferred from neutron diffraction studies in $R_{2} \mathrm{CuO}_{4} \cdot{ }^{21}$ For example, in $\mathrm{Pr}_{2} \mathrm{CuO}_{4}$ about $0.08 \mu_{B}$ of $\mathrm{Pr}$ moments are induced by $\mathrm{Cu}$ moments with $0.4 \mu_{B},{ }^{21}$ with a non-collinear spin structure in the $a b$ planes for both sublattices. ${ }^{22}$ In a mean-field treatment, the $\mathrm{Cu}$ ions exert an effective magnetic field on the $\mathrm{Pr}$ ions so that the moment size of the Pr ions is given by

$$
\left\langle M_{\mathrm{Pr}}\right\rangle \sim \chi_{a b} J\left\langle M_{\mathrm{Cu}}\left(H_{z}\right)\right\rangle,
$$

where $J$ is the $\mathrm{Cu}^{2+}-\mathrm{Pr}$ interaction energy and $\left\langle M_{\mathrm{Cu}}\left(H_{z}\right)\right\rangle$ is the field-induced $\mathrm{Cu}$ moments. ${ }^{21}$ Thus, we can expect an additional field $\Delta B_{z} \simeq A_{f}\left\langle M_{\mathrm{Pr}}\right\rangle$, which is induced by the polarized $\mathrm{Cu}$ ions in the superconducting phase. Because of the non-diagonal hyperfine coupling $A_{f}$ between muons and Pr ions, a small in-plane polarization of Pr ions can lead to a sizable magnitude of hyperfine field along the $c$-axis on the nearby muons. Our estimation indicates that about $0.01 \mu_{B}$ of $\operatorname{Pr}$ moments, which may be induced by $0.05 \mu_{B}$ of $\mathrm{Cu}$ moments, is enough to account for the amplitude of $\Delta B_{z}$.

It is interesting to note that the strong influence of the superconducting phase is observed at as low an external field as $10^{2}$ Oe, where the density of magnetic vortices is very small (their distance being $\sim 4 \times 10^{3} \AA$ ). Considering that most of the implanted muons are probing the sample outside the vortex cores in this low field range, we can conclude that the origin of the enhanced muon Knight shift associated with the superconducting phase is not confined in the vortex cores. Thus, it is highly presumable that the small polarization of Pr ions (and of $\mathrm{Cu}$ ions) is present in the entire volume of the specimen under an external field, as suggested by the neutron diffraction. This might be in favor of the quantum critical point scenario in understanding the competition between AF and superconducting phases. ${ }^{23}$

Finally, we note that additional work is clearly needed to elucidate the origin of unconventional hyperfine coupling between muon and Pr ions, including that on the possible role of muon itself as a source of perturbation.

In summary, we have shown that the muon Knight shift, which is anomalously proportional to the in-plane susceptibility (perpendicular to the field direction), is strongly enhanced by the occurrence of superconductivity, changing its sign from positive to negative around $\sim 1 \mathrm{kOe}$ with increasing field. This result can be understood by considering weak in-plane polarization of $\mathrm{Pr}$ ions, which are polarized by field-induced $\mathrm{Cu}$ moments via superexchange interaction. It also suggests strongly that there is a uniform weak polarization of $\mathrm{Cu}$ moments induced by an external field as low as $10^{2}$ Oe in the superconducting phase of $\mathrm{Pr}_{0.89} \mathrm{LaCe}_{0.11} \mathrm{CuO}_{4-y}$, irrespective of vortex cores in the mixed state. This extraordinary sensitivity of $\mathrm{CuO}_{2}$ planes to a magnetic field will provide a strong criterion for identifying the true electronic ground state of $n$ type cuprates.

We wish to thank the staff of TRIUMF for their technical support during the experiment. This work was partially supported by a Grant-in-Aid for Scientific Research on Priority Areas and a Grant-in-Aid for Creative Scientific Research by the Ministry of Education, Culture, Sports, Science and Technology, Japan

1) P. Monthoux, A. V. Balatsky, and D. Pines, Phys. Rev. B 46, 14803 (1992).

2) Y. Suzumura, Y. Hasegawa, and H. Fukuyama, J. Phys. Soc. Jpn. 57, 2768 (1988)

3) J. Zaanen, O. Y. Osman, and W. van Saarloos, Phys. Rev. B 58, R11868 (1998).

4) S. Katano, M. Sato, K. Yamada, T. Suzuki, and T. Fukase, et al., Phys. Rev. B 62, R14677 (2000).

5) B. Lake, G. Aeppli, K. N. Clausen, D. F. McMorrow, K. Lefmann, N. E. Hussey, N. Mangkorntong, M. Nohara, H. Takagi, T. E. Mason, and A. Schröder, Science 291, 1759 (2001).

6) B. Lake, H. M. Ronnow, N. B. Christensen, G. Aeppli, K. Lefmann, D. F. McMorrow, P. Vorderwisch, P. Smeibidl, N. Mangkorntong, T. Sasagawa, M. Nohara, H. Takagi, and T. E. Mason, Nature 415, 299 (2002).

7) V. F. Mitrović E. E. Sigmund, M. Eschrig, H. N. Bachman, W. P. Halperin, A. P. Reyes, P. Kuhns, and W. G. Moulton, Nature 413, 501 (2001).

8) K. Kakuyanagi, K. Kumagai, and Y. Matsuda, Phys. Rev. B 65, 060503 (2002).

9) J. E. Hoffman, E. W. Hudson, K. M. Lang, V. Madhavan, S. H. Pan, H. Eisaki, S. Uchida, and J. C. Davis, Science 295, 446 (2002).

10) R. I. Miller, R. F. Kiefl, J. H. Brewer, J. E. Sonier, J. Chakhalian, S. Dunsiger, G. D. Morris, A. N. Price, D. A. Bonn, W. H. Hardy, and R. Liang, Phys. Rev. Lett. 88, 137002 (2002).

11) G.-q. Zheng, T. Sato, Y. Kitaoka, M. Fujita, and K. Yamada, Phys. Rev. Lett. 90, 197005 (2003).

12) K. Yamada, K. Kurahashi, T. Uefuji, M. Fujita, S. Park, S.-H. Lee, and Y. Endoh, Phys. Rev. Lett. 90, 137004 (2003).

13) H. J. Kang, Pengcheng Dai, J. W. Lynn, M. Matsuura, J. R. Tompson, Shou-Cheng Zang, D. N. Argyriou, Y. Onose, and Y. Tokura, Nature 423, 522 (2003).

14) M. Matsuura, Pengcheng Dai, H. J. Kang, J. W. Lynn, D. N. Argyriou, Y. Onose, and Y. Tokura Phys. Rev. B 69, 104510 (2004).

15) J. E. Sonier, K. F. Poon, G. M. Luke, P. Kyriakou, R. I. Miller, R. Liang, C. R. Wiebe, P. Fournier, and R. L. Greene, Phys. Rev. Lett. 91, 147002 (2003).

16) M. Fujita, T. Kubo, S. Kuroshima, T. Uefuji, K. Kawashima, and K. Yamada Phys. Rev. B 67, 014514 (2003).

17) M. Fujita et al., cond-mat/0311269.

18) R. Kadono, K. Ohishi, A. Koda, W. Higemoto, K. M. Kojima, S. Kuroshima, M. Fujita, and K. Yamada, J. Phys. Soc. Jpn. 72, 2955 (2003).

19) G. M. Luke, L. P. Le, B. J. Sternlieb, Y. J. Uemura, J. H. Brewer, R. Kadono, R. F. Kiefl, S. R. Kreitzman, T. M. Riseman, C. E. Stronach, M. R. Davis, S. Uchida, H. Takagi, Y. Tokura, Y. Hidaka, T. Murakami, J. Gopalakrishnan, A. W. Sleight, M. A. Subramanian, E. A. Early, J. T. Markert, M. B. Maple, and C. L. Seaman, Phys. Rev. B 42, 7981 (1990).

20) L. P. Le, G. M. Luke, B. J. Sternlieb, Y. J. Uemura, J. H. Brewer, T. M. Riseman, D. C. Johnston, L. L. Miller, Y. Hidaka, and H. Murakami, Hyperfine Interact. 63. 279 (1990).

21) M. Matsuda, K. Yamada, K. Kakurai, H. Kadowaki, T. R. 
Thurston, Y. Endoh, Y. Hidaka, R. J. Birgeneau, M. A. Kastner, P. M. Gehring, A. H. Moudden, and G. Shirane Phys. Rev. B 42, 10098 (1990).
22) S. Skanthakumar, J. W. Lynn, J. L. Peng, and Z. Y. Li, Phys. Rev. B 47, 6173 (1993).

23) S. Sachdev, Rev. Mod. Phys. 75, 913 (2003). 